\title{
Die Geschichte der Stadt und der Ausgrabungen.
}

Das Schicksal der Stadt Fompeji läßt sich niłt dem eines Mannes vergleichen, der erst nach seinem Tode berühmt geworden ist.

Im Altertum war es eine Stadt wie hundert andere, deren Einwohnerzahl 20000 nicht überstieg. Im Rahmen des römischen Reiches stellte sie nichts Besonderes dar. Wir haben in der Tat kaum zwanzig antike Zitate, in denen Pompeji erwähnt wird und selbst in diesen geschieht das nur nebenbei. Aber sie sind uns sehr wichtig, denn sie verhelfen uns wenigstens zu einiger Kenntnis der Geschichte der Stadt und ihre Monumente. Cicero führt die Stadt im Jahre 63 v. Chr. unter denen auf, dic am besten eingerichtet waren, und vergißt nicht hinzuzufügen, $\mathrm{da} B$ sie schon eine lange und mühselige Geschichte hinter sich habe. Die Geschichte der Stadt vor dem II. Jahrhundert können wir nur in ganz großen Linien rekonstruieren.

Der Platz, auf dem die Stadt entstand, hat viele für die Gründung und Entwicklung einer Ansiedlung günstige Eigenschaften. Die ersten Bewohner ließen sich auf einem $\mathrm{Hügel}$ vulkanischen Ursprungs nieder, der an zwei Seiten steil zur Ebene abfiel. Auch die Nähe der Mündung eines in damaliger Zeit schiffbaren Flusses (Sarno) war von Bedeutung. So erfüllte die Lage der Stadt alle für die Antike notwendigen strategischen und verkehrspolitischen Bedingungen.

Die Gründer von Pompeji waren die Osker, ein $Z_{\text {weig }}$ der italischen Rasse. Ihre Sprache ist immer noch nicht ganz erforscht und in fast jeder Inschrift findet man Worte, deren Bedeutung zweifelhaft ist. Wir werden darauf gelegentlich zurückkommen. Der Name "Pompei" wird von den meisten Forschern auf das oskische Wort "pompe", das fünf bedeutet, zurückgeführt. So daB also der Name Pompejus 
gleich bedeutend mit dem lateinischen Quintus sein würde. Vielleicht erhielt die Stadt ihren Namen von einem Herrengeschlecht, einer "Gens", das kurze Zeit die politische Macht besaB.

Das älteste Gebäude in Pompeji ist der sog. griechische Tempel (s. S. 196), dessen GrundriB dem der unteritalischen Tempel des VI. Jahrhunderts verwandt ist. Der griechische EinfluB kam nach Pompeji von Cumae einerseits und Paestum andererseits. Wir werden Gelegenheit haben von ihm zu sprechen. Bis noch vor kurzem wurde die Bedeutung des griechischen Einflusses sehr übertrieben, jetzt verneinen einige Forscher ihn ganz. Die Wahrheit wird, wie immer, in der Mitte liegen.

Wie ganz Campanien stand auch Pompeji eine Zeitlang unter etruskischer Herrschaft, die aber ohne tiefergehenden EinfluB blieb. Die Etrusker bildeten nur eine dünne aristo. kratische und militärische Oberschicht. Schon um die Mitte des V. Jahrhunderts war die etruskische Herrschaft zu Ende. Wenig später brach das Gebirgsvolk der Samniter in die Ebene ein und es gelang ihm, sich einen Weg zum Meere zu bahnen durch die Eroberung einiger Küstenstädte. Von den Streitigkeiten $z$ wischen den Eindringlingen und den Bewohnern der Ebene profitierte das damals schon mächtige Rom. Es gelang ihm, sich das ganze Gebiet in dres Kriegen (342-290) zu unterwerfen. In den Berichten des Titus Livius über diese Kriege finden wir bei ihm die einzige $\mathrm{Er}$ wähnung der Stadt. Von nun an teilte Pompeji das Schicksal aller campanischen Städte. Während des zweiten punischen Krieges ergriff es Hannibals Partei und machte gemeinsame Sache mit den Aufrührern.

Im Jahre 83 v. Chr. wurde die Stadt von einem römischen Heer unter Sullas Führung belagert. Ueber die letzten Begebenheiten des Krieges wissen wir nichts Näheres. Wir hören nur, daB im Jahre $80 \mathrm{v}$. Chr. eine größere Anzahl von Veteranen in der Stadt angesiedelt wurden, die seitdem Colonia Veneria Cornelia heiBt. Mit diesem Zeitpunkt beginnt die eigentliche römische Epoche der Stadt. Die unvermeidlichen Reibungen $z$ wischen den alten und neuen Bewohnern der Stadt hatten keine ernstlichen Folgen, denn die Verwaltung der Stadt leitete mit großem Geschick und Takt Publio Sulla, der Neffe des Diktators. Von seiner Umsicht und seinen Fähigkeiten berichtet verschiedentlich der unerschöpfliche Cicero. 
Unter der römischen Herrschaft blühte die Stadt von neuem auf. Jetzt machte sich vor allem ihre für den da. maligen Handel günstige Lage geltend. In der Kaiserzeit vollzieht sich dann immer mehr eine stärkere Annäherung an Rom. Mehrere Fürsten werden zu Schutzherren der Stadt ernannt und ihnen Standbilder errichtet. Ein großes Unglück suchte die Stadt im Februar des Jahres 63 n. Chr. heim. Um den Eindruck wiederzugeben, den diese Katastrophe machte, lassen wir einen Brief des Seneca hier folgen: „Bester Lucilius! Wir haben gehört, daß Pompeji, eine bedeutende Stadt Campaniens, die an einer anmutigen Bucht am Meer liegt, wo die Ufer von Surrentum und Stabiae und das von Herculaneum zusammentreffen, von einem Erdbeben zerstört wurde. Auch die umliegenden Gebiete wuiden sehr verwüstet. Es geschah zur Winterszeit, die nach den Berichten unserer Voriahren von dieser Gefahr frei sein soll. Das Erdbeben erfolgte an den Nonen des Februar in dem Consulatsjahr des Regulus und des Vergmius. Diesmal suchte das Beben Campanien mit großen Verwüstungen heim. Und die allgemeine Ueberraschung war um so größer, als Campanien, das $z$ war nie von diesen Naturereignissen ganz frei ist, bisher ummer ohne großen Schaden davon gekommen. Auch von Herkulaneum wurde ein Teil zerstört und die Colonie Nuceria erlitt erhebliche Beschädigungen. Neapel hat viel an Privathäusern verloren, aber nichts an öffentlichen Gebäuden und wurde verhältnismäBig leicht von der Katastrophe betroffen. Viele Landhäuser stürzten ein oder wurden erheblich beschädigt. Statuen zerbrachen. Viele Menschen irrten nachher mit verstörtem Geist umher und ihrer selbst nicht mächtig."

Mit großem Eifer machten sich die Bewohner nach dieser Katastrophe an den Wiederaufbau. Aber sie sind mit ihrer Arbeit nicht fertig geworden. Das große Erdbeben vom Jahre 79 überraschte sie vollständig. Von dem furchtbaren Eindruck dieser Katastrophe geben uns die beiden Briefe des Plinius, die wir hier folgen lassen ein erschütterndes Zeugnis.

\section{Plinius an Tacitus.}

Du ersuchst mich. Dir über das Ende meines Oheims zu schreiben, um es der Nachwelt desto getreuer überliefern zu können. Er befand sich zu Misenum, als Kommandant der Flotte. Am 23. August ungefähr um ein Uhr nachmittags, meldete ihm meine Mutter, es zeige sich eine 
Wolke von außergewöhnlicher Größe und Gestalt. Er hatte sich in der Sonne ergangen, dann kalt gebadet, auf dem Lager liegend etwas genossen und studierte. Er forderte seine Sandalen und stieg auf eine Anhöhe, von wo aus man die wunderbare Erscheinung am besten beobachten konnte. Die Wolke stieg aut - von we!chem Berge, war aus der Ferne nicht genau zu unterscheiden; daB es der Vesuv war, erfuhr man erst nachher - sie sah ihrer ganzen Gestaltung nach einem Baume und zwar einer Fichte ähnlich. Sie hob sich nämlich wie mit einem sehr langem Stamme empor und zerteilte sich dann in verschiedene Aeste; wahrscheinlich verlor sie sich in die Breite, weil sie durch den frischen Luftzug zuerst in die Höhe getrieben, dann, als dieser an Kraft abnahm oder durch ihr eigenes Gewicht, wieder herabgedrückt wurde. Sie erschien zuweilen glänzend weiß, dann wieder schmutzig und fleckig, je nachdem sie Erde oder Asche mit sich führte. Die Sache erschien ihm als einem gelehrten Manne wichtig und einer näheren Untersuchung wert. Er gab Befehl eine Jacht in Bereitschaft zu setzen; mir stelite er es frei ihn zu begleiten, wenn ich Lust hätte. Ich erwiderte, ich wolle lieber studieren, und zufälligerweise hatte er mir selbst etwas $\mathrm{zu}$ schreiben aufgegeben. Er trat eben aus dem Hause, als er ein Schreiben erhielt. Das Schiffspersonal zu Resina, durch die drohende Gefahr erschreckt - denn das Oertchen lag am FuB des Berges und man konnte sich nur zu Schiffe retten bat ihn, sie doch einer so großen Gefahr zu entreißen. Er änderte nun seinen Plan, und was er als Gelehrter begonnen hatte, verfolgte er mit Heldenmut. Er läßt Vierruderer unter Segel gehen, geht selbst an Bord, um nicht nur Resina, sondern auch noch vielen anderen - denn die Küste war ihrer Anmut wegen sehr bevölkert - Hilfe zu bringen. Er eilt dahin, von wo andere entfiiehen und steuert in gerader Richtung der Gefahr zu, so sehr aller Furcht bar, daB er alle Bewegungen, alle Gestalten dieser unglückseligen Erscheinungen, wie sie sich seinen Augen darstellten, diktierte und aufzeichnen lieB. Bereits fiel Asche in die Schiffe, und je näher er hinzukam, desto heißer und dichter, schon auch Bimssteine und schwarzes, ausgebranntes und vom Feuer zerbröckeltes Gestein. Jetzt zeigte sich plötzlich eine Untiefe, und der Schutt vom Berge her machte das Ufer unzugänglich. Er bedachte sich eine kurze Weile, ob er zurücksteuern solle; bald aber sagte er zu dem Steuermann, welcher ihm dieses anriet: „Frisch gewagt ist 
halb gewonnen; fahre zu Pompejanus!" Dieser war zu Stabiae, aut der entgegengesetzten Seite der Bucht; denn das Meer tritt hier in das allmählich sich krümmende und herumziehende Ufer hinein. Obgleich hier die Gefahr noch nicht so nahe war, hatte man sie dennoch vor Augen, und wenn sie zunahm, war sie ganz nahe. Pompejanus hatte daher sein Gepäck in sein Schiff schaffen lassen, fest entschlossen, zu fliehen, so bald der widrige Wind sich gelegt haben würde. Als mein Oheim, welchem der Wind im höchsten Grade günstig war, gelandet war, umarmte er den Zitternden, tröstet und ermuntert ihn; und um dessen Furcht durch eigene Furchtlosigkeit zu lindern, ließ er sich ins Bad bringen, legte sich nach dem Bade $z u$ Tische; speiste heiter oder, was ebenso groß ist, mit der Miene eines Heiteren. Indessen leuchteten aus dem Berge Vesuv an mehreren Stellen weithin sich ausbreitende Flammen und hohe Feuersäulen, deren Glanz und Helle, durch das Dunkel der Nacht noch erhöht wurde. Um der Furcht zu steuern, sagte er, es seien dies die Bauernhöfe, welche von den Landleuten in der Angst verlassen und dem Feuer preisgegeben worden seien und jetzt leer daständen und brennen. Hierauf begab er sich zur Ruhe und genoß wirklich eines ganz festen Schlafes; denn sein Atemholen, das wegen seines schweren Körpers etwas schwer und schnarchend war, wurde von den Leuten, welche sich vor der Tür seines Gemaches befanden, vernommen. Allein der Hof, von dem aus der Zugang in das Zimmer führte, war bereits von mit Bimssteinen vermischter Asche so hoch angefült, daB ihm, wenn er noch länger in dem Schlafgemach verweilt hätte, das Herauskommen nicht mehr möglich gewesen wäre. Man weckte ihn; er ging heraus und begab sich zu Pompejanus und den anderen, die wachgeblieben waren. Sie berieten sich gemeinschaftlich ob sie im Hause bleiben oder im Freien herumgehen wollten. Denn die Häuser wankten von den vielen und heftigen Erdstößen, und wie wenn sie aus ihrem Grunde gehoben wurden, schienen sie sich bald von der Stelle $z u$ bewegen, bald wieder an der vorigen zu stehen. Andererseits fürchtete man im Freien das Herab. fallen der, wenngleich leichten und ausgebrannten, Bimssteine. Doch wählte man bei Vergleichung der Gefahren das letztere. Und zwar siegte bei ihm ein Grund über den anderen, bei den anderen eine $F$ urcht über die andere. Sie banden sich mit Tüchern Kissen um den Kopf; dies diente zum Schutz gegen den Steinregen. Schon war es ander- 
wärts Tag, dort war es Nacht, dichter und schwärzer als alle Nächte, doch wurde dieselbe durch Fackeln und sonstige Beleuchtung erhellt. Man beschloß, an das Gestade hinauszugehen, um aus der Nähe zu sehen, ob das Meer befahrbar wäre; dieses blieb aber immer wild und ungestüm. Hier legte er sich auf ein hingebreitetes Tuch, und verlangte $z u$ wiederholten Malen frisches Wasser und trank. Nun trieben die Flammen und der den Flammen vorausgehende Schwefelgeruch die anderen in die Flucht: ihn machten sie nur munter. Aut zwei Sklaven gestüzt erhob er sich, sank aber plötzlich nieder; wie ich vermute, hatte ihm der dichte Dampf den Atem gehemmt und den Magen gesperit, der bei ihm ohnedies von Natur schwach und eng war und häufige Krämpfe veranlaBte. Als es wieder Tag wurde -- es war der dritte nach dem, welchen er zuletzt erblickt hatte fand man seinen Körper unversehrt, ohne Verletzung und mit derselben Bekleidung, welche er angehabt hatte; das Aussehen seines Körpers war mehr das eines Schlafenden, als eines Toten.

\section{Brief des Plinius an Tacitus.}

Nachdem mein Oheim fortgegangen war, verbrachte ich die übrige Zeit mit Studieren; denn dies war der Grund meines Zurückbleibens. Hierauf badete ich, speiste und schlief, aber unruhig und nur kurz. Schon mehrerc Tage vorher hatte sich ein großes Erdbeben spüren lassen, was aber eben zu keiner groBen Furcht AnlaB gab, weil dies in Campanien etwas Gewöhnliches ist; in jener Nacht aber wurde es so stark, daB alles nicht nur sich zu bewegen, sondern zusammenzufallen schien. Meine Mutter stürzte in mein Schlafzimmer; ich stand eben auf, um meinerseits sie zu wecken, wenn sie etwa noch schliefe. Wir setzten uns in den Hof des Hauses, welcher das Meer von den Häusern durch einen mäßigen $Z$ wischenraum trennte. Es war schon 6 Uhr morgens und noch war der Tag nicht recht da lind - wenn ich so sagen darf — träge im Werden; da die umliegenden Gebäude bereits heftige Erschütterungen erlitten hatten, so war, da der Platz zwar frei, aber doch beschränkt war, die Furcht vor einem Einsturz groB und wohl begründet. Jetzt erst schien es uns geraten, die Stadt zu verlassen. Die bestürzte Menge folgte uns nach; sie zog - was in der Angst für Klugheit gilt - fremden Rat dem eigenen vor, und in dichte Haufen geschart, drückte und trieb sie uns zum Gehen vorwärts. Als wir die Häuser hinter uns hatten, 
blieben wir stehen. Vieles gab es auch hier, was uns staumen, was uns erschrecken machte. Denn die Wagen, welche wir hatten hinausführen lassen, wurden auf völlig freiem Felde hin und her geworfen, und selbst, als man Steine unterlegte, blieben sie nicht fest stehen. Und auch das Meer gewährte einen Anblick, als ob es sich selbst verschlänge und durch die Erderschütterung zurückgetriehen würde. Wenigstens war das Gestade weiter vorgerückt und es befanden sich darauf viele aut dem trockenen Sande zurückgebliebene Seegeschöpfe. Aut der anderen Seite zerbarst eine Wolke, aus welcher geschlängelte Feuermassen nach allen Seiten herauszuckten und entlud sich in langen Flammenstrahlen, welche Blitzen ähnlich, aber weit größer waren ..... Schon fiel Asche, doch nicht stark. Ich blickte zurück: ein dicker Dampf kam hinter uns her, der uns gleich einem aut die Erde sich ergieBenden Strome nachzog .... Kaum hatten wir uns gesetzt, als es Nacht ward, nicht so, wie wenn der Mond nicht scheint oder der Himmel umwölkt ist, sondern, wie wenn man an verschlossenen Orten das Licht auslöscht. Man hörte Geheul von Weibern, Gewimmer von Kindern, Geschrei von Männern: die einen riefen ihre Eltern, andere ihre Kinder, wieder andere ihren Gatten, welche sie an ihren Stimmen erkannten; diese bejammerten ihr eigenes Geschick, jene das der Ihrigen; auch waren darunier solche, welche sich aus Furcht vor dem Tode den Tod wünschten .... Es wurde wieder ein wenig hell, was uns nicht wie der wirkliche Tag, sondern wic der Vorbotc des nahenden Feuers vorkam, doch blieb dieses Feuer in ziemlicher Entfernung; hierauf wurde es wieder finster, und es fiel wieder dichte Asche in großer Menge. Wir mußten zu wiederholten Malen aufstehen und sie abschütteln, sonst wären wir verschüttet und von ihrer Last erdrückt worden.... Endlich löst sich jener dichte Dampf in eine Art Nebel oder Rauch auf; es wurde wirklicher Tag, auch die Sonne glänzte hervor, aber nur ganz blaß, wie dies bei einer Sonnenfinsternis zu sein pflegte."

Der Kaiser Titus ernannte eine eigene, aus Senatoren bestehende Kommisssion, die die Aufgabe bekam, für den Wiederaufbau der verwüsteten Gebiete Sorge zu tragen. Ein Wiederaufbau aber war unmöglich: Herkulaneum war unter einer dicken verhärteten Schlammschicht begraben und von Pompeji ragten nur die höchsten Spitzen einiger Gebäude aus dem Aschenmeer. Dringend not aber tat die Hilfe den unglücklichen Bewohnern der Städte, denen es gelungen war, sich in Sicherheit zu bringen. Und die meisten müssen 
doch entkommen sein, denn bei den Ausgrabungen hat man vcrhälınismäßig wenig Skelette gefunden. Mit der Unterstützung der Kommission haben dann die Geretteten versucht, ihr Eigentum wiederzuerlangen, indem sie tiefe Löcher und Stollen in die Schuttmassen gruben. Dabei haben sie manches gerettet. Aber auch Plünderer fanden sich ein. Beider Spuren sind die Forscher bei den Ausgrabungen oft begegnet. Ein Plünderer scheint auch der Mann gewesen zu sein, dessen Skelett man mit einer Laterne in einem Loch in einer Mauer fand und $z$ war in der Aschenschicht. DaB einige Bewohner nach der Katastrophe wieder in die Stadt gelangt sind, schließt man aus einigen $G \mathrm{raf} f \mathrm{it}$, die man sonst nicht erklären kann. Nachdem man soviel wie möglich weggeschafft hatte, wurden die Ruinen verlassen. Das weitere Schicksal der Einwohner von Pompeji ist nicht bekannt. Eine Inschrift, die in der fernen Dobrudscha gefunden wurde, an der Küste des heutigen Rumänien, erzählt von einem Flüchtling aus Pompeji, der im Kampfe gegen die Barbaren fiel.

Irgendeine Erinnerung, daß unter der Asche cine ganze Stadt begraben sei, lebte in dem Gedächtnis der Umwohner noch Jahrhunderte lang fort. Die mit Schuttmassen bedeckte Anhöhe, unter der Pompeji verborgen lag, erhielt den Namen „,cività". Um so verwunderlicher ist es, daB der Architekt Domenico Fontana, als er bei dem Bau eines Wasserleitungsstoliens, der durch die ganze Stadt geht und den wir noich an einigen Stellen heute sehen können, einen Stein mit der Inschritt, in der Pompeji erwähnt wurde, nicht verstanden hat, dab es sich hier um die bei Plinius erwähnte Stadt handele. Das war für dic Archäologic cin großes Glück, denn wenn Pompeji schon damals entdeckt worden wäre, so wäre für uns wohl kaum etwas zum Entdecken geschweige zum Bcwundern übriggeblieben. Und trotzdem kann man noch sagen, daB Pompeji zu früh entdeckt wurde.

Die Ausgrabungen begannen am 30. März 1748. Durch einen Zufallsfund, den einige Weinbergsarbeiter gemacht hatten, wurde die Aufmerksamkeit des Ingenieurs Alcubierre, der sich seit 10 Jahren der Ausgrabung Herkulaneums gewidmet hatte, auf diese Stätte gelenkt und er setzte seine Forschungen hier fort in der Meinung, das antike Stabiae $\epsilon$ ntdeckt zu haben. Für seine Arbeit standen ihm mehrere Sträflinge zur Verfügung und am Ende jeder Woche hatte er Rechenschaft über seine Tätigkeit und die Funde abzulegen. lhm schien Pompeji eine Art Bergwerk, das ausgebeutet 
werder mußte. Und zu seiner Zeit wurde aus den Häusern alles fortgeschleppt und ins Museum gebracht, was sich irgend fortragen lieb. Auf diese Weise wurde manches Fresko zerstört. Erst als man nach etwa fünfzehnjähriger Ausgrabungstätigkeit die Statue des Suedius Clemens fand, tauchten $Z$ weifel auf, ob man es hier mit Stabiae oder Pompeji zu tun habe. Im Jahre 1764 beklagt Winkelmann es sehr, daB man für eine derartig bedeutende Ruinenstätte nur acht Arbeiter zur Verfügung habe. Fünf Jahre später äußert sich der Kaiser von Oesterreich, Joseph II., seinem jugendlichen Schwager, dem König Ferdinand, gegenüber sehr entrüstet über die Tatsache, da $B$ nur 30 und nicht 30000 Arbeiter, wie er es für richtig hielte, bei den Ausgrabungen beschäftigt würden. Und außerdem scheint es ihm fraglich, ob es richtig sei, alle Gegenstände aus der Stadt fortzuschaffen.

Die politischen Ereignisse am Ende des 18. und Anfang des 19. Jahrhunderts waren nicht ohne Einfluß auf die Ausgrabungen. Ein napoleonischer General, Championnet, hat z. B. zwel Häuser ausgegraben, die noch heute seinen Namen tragen.

Mit großem Erfolg arbeitete man in Pompeji in den Jahren 1806-1815 unter der Regierung Joseph Bonapartes und Joachim Murats. In dieser Zeit kamen die GräberstraBc mit der Villa des Diomedes, das große Theater, die Basiliika, ein Teil des Forums und einige Häuser ans Licht. Der Leiter der Ausgrabungen war damals Arditti, der bei seinen Unternehmungen ganz systematisch vorging. Unter den Bourbonen hingegen schritten die Arbeiten weniger gut fort und wurden auch weniger sorgfältig gemacht. Das ist um so mehr zu beklagen, als in dieser Zeit die schönsten Häuser gefunden wurden.

Die Vereinigung des Königreichs Neapel mit Italien bedeutete eine günstige Wendung für die pompejanischen Ausgrabungen. Jetzt trat Giuseppe Fiorelli an die Spitze der Arbeiten und mit ihm beginnt eine neue Epoche der Forschungen in Pompeji. Als er die Leitung übernahm, boten die Ruinenstätten einen beklagenswerten Anblick. Die dringendste Aufgabe war, die ungeheuren Erdhaufen, die von den früheren Ausgrabungen her in den schon ausgegrabenen Straßen lagen, fortzuschaffen. Erst dann konnten die eigentlichen Arbeiten beginnen. Fiorellis Gedanke war es, daß Pompeji nicht als ein Bergwerk für Antiken zu betrachten sei, sondern daB die Stadt an sich schon ein Museum sei und daß für die Archäologen jeder Gegenstand von gleicher Be- 
deutung ist, ganz gleich, aus welchem Material er gemacht ist. Mit Fiorelli beginnt also die systematische Ausgrabung. Unter anderen wird von nun an von oben nach unten bis zu einer bestimmten Tiefe gegraben. Entsprechend dem Gedanken, daB Pompeji ja selbst ein Museum sei, ordnete Fiorelli an, daB die Mehrzahl der Gegenstände an ihrem Platz $z \mathrm{u}$ bleiben hätten und daB nur die Bronzestatuen wegen ihres Wertes fortgeschafft und durch Kopien ersetzt werden sollten. Auch die Fresken, die verderben hätten können, wurden ins Museum gebracht. 1863 gelang es Fiorelli, die Abgüsse einiger Toten wiederzugewinnen, indem er die Höhlung, die der Körper in dem allmählich verhärteten Aschenbre! hinterlassen hatte, mit Gips ausgoß. Auf diese Werse erhielt man eine Reihe von Abgüssen, von denen etwa zehn, darunter der eines Hundes sich im kleinen Lokalmuseum befinden. Andere hat man in dem Hause gelassen, in dem man sie gefunden hat, neuerdings auch einige in der Casa cryptoportica in den Nuovi Scavi. Diese Abgüsse bilden den besten Kommentar zu den Briefen des Plinius. Auch für die verschwundenen Gegenstände aus $\mathrm{Holz}$ usw., die Hohlräume in der Aschenmasse hinterlassen haben, hat man dieses Verfahren angewandt, z. B. für die Türen, Schränke und Stühle. Nachdem Fiorelli Generalverwalter der Altertümer Italiens geworden war, arbeiteten seine Nachfolger in seiner Weise weiter. Seine Systeme wurden ausgebaut und in immer großartigerer Weise angewendet. Schließlich wurden, als man im Jahre 1895 die Casa dei Vettii fand, nicht nur die Skulpturen und Fresken an ihrem Ort gelassen, sondern man versuchte auch den Garten wiederherzustellen. Das gleiche geschah in den Häusern, die man in den folgenden Jahren fand, wie z. B. in der Casa degli amorini dorati und delle Nozze d'argento und in all den Häusern zu Seiten der Via dell'Abbondanza, die zu der Zone der sog. Nuovi Scavi gehören.

Leider besteht keine Hoffnung, $\mathrm{da} B$ in Pompeji noch andere öffentliche Gebäude, außer den schon entdeckten, gefunden werden könnten, höchstens Badeanstalten wird man noch finden.

Ehe man den Rundgang durch die Stadt beginnt, wird es nützlich sein, einen Blick auf den Stadtplan zu werfen, damit man sich nachher leichter orientiert. Die Prinzipien, nach denen die Straßen angelegt sind, sind die gleichen wie in allen italischen Städten. Wir haben zwei sich kreuzende Hauptachsen, den Cardo von Norden nach Süden und den 
Decumanus, der von Osten nach Westen geht. Natürlich war die Ausdehnung der Stadt in den ersten Jahrhunderten ihres Bestehens viel geringer wie zur Zeit der Verschüttung. Die Kreuzung der beiden Achsen befand sich nicht an der heutigen Stelle. Der Cardo ist die heutige Via di Stabiae, die vom Stabianertor zum Vesuvtor führt und der Decumanus die Via di Nola, die etwas westlich vom Nolanertor beginnt und am Vicolo del Farmacista vor einem noch nicht ganz ausgegrabenen Hause endet. Die ältere Kreuzung lag dort. wo sich jetzt das Forum befindet, das damals kleiner war, wie das, was wir jetzt vor uns sehen. $Z u$ dieser Zeit bildeten den Cardo die heutige Via delle scuole und Via del Foro und den Decumanus die Via della Marina und die Via dell'Abbondanza, die das Forum an der Südseite begrenzten. $\mathrm{DaB}$ die Hauptachsen sich nicht genau in rechten Winkel treffen, sondern einen spitzen Winkel bilden, lag an den Bodenverhältnissen. Fast rechtwinklig treffen sich die Straßen nur in der Regio VI im Nordwesten, wo sich die schönsten Häuser der Stadt befinden.

Im Unterschied $z u$ dieser regelmäßigen Anlage fällt uns der südlich von ihm gelegene Stadtteil mit den unregelmäßigen und engen Straßen auf. In diesem Teil liegen die meisten öffentlichen Gebäude, woraus man vielleicht schließen kann, daß dieser Teil der ältere ist und die regelmäßige Anlage einer späteren Vergrößerung der Stadt zuzuschreiben ist. In vielen Städten, die einen plötzlichen Aufschwung nahmen, kann man eine derartige Entwicklung beobachten, so $z$. B. in Süditalien in Tarent. Gegen die spätere Datierung dieses regelmäßig angelegten Quartieres haben einige Forscher den Fund einer dorischen Säule in ihm angeführt; wir werden darüber an anderer Stelle sprechen (s. S. 63).

Die Instandhaltung der Bürgersteige, die in allen Straßen vorhanden waren, lag den Hausbesitzern ob. Dadurch erklärt sich auch die große Uneinheitlichkeit wie z. B. bei den Bürgersteigen vor der Casa di Trittolemo (VII. 7 1-12). hier 1st das Pflaster mit einem reizvollen ornamentalen Muster geschmückt. Die Bürgersteige sind auch verschieden hoch. Die Straße selbst ist von Zeit zu Zeit von einem oder mehreren großen ovalen Steinen unterbrochen, die so hoch wie die Bürgersteige sind und die dazu dienten, den Uebergang von einem Bürgersteig zum anderen zu erleichtern, damit man während des Regens nicht allzu nasse Füße bekam und auch 
nicht immer auf und ab klettern mußte. Oft hat man zu diesem $Z$ weck Reste von Kapitellen oder Mühlsteine benutzt. Sie bedeuteten zwar eine Bequemlichkeit für die Menschen, aber nicht für die Tiere, die vor schwere Karren gespannt waren und über diese Steine herüber mußten. Deutlich erkennt man $z$ wischen den Steinen die tiefen Spuren der Wagenräder.

Die schönste Straße der Stadt, die Strada del Foro erreicht eine Breite von 9 Meter. Alle Straßen Pompejis sind mit denselben unregelmäßigen rechteckigen Steinen gepflastert. Genau weiß man nicht, wann die Straßen Pompejis zuerst gepflastert wurden. Den einzigen chronologischen Anhaltspunkt bietet eine Inschrift auf einem Pflasterstein, in der der Monat Quinctilis, der unserm Juli entspricht, erwähnt wird. Später nach der Heroisierung Julius Cäsars nannte man ihn Julius.

Besonders großes Gewicht legte man in dieser Zeit auf die Wasserversorgung der Städte. Die erste Wasserleitung wurde in Pompeji zwischen dem zweiten punischen Krieg (200) und dem Bundesgenossenkrieg (90-80) erbaut, einen genaueren Zeitpunkt zu bestimmen, ist nicht möglich, da wir keine festen Anhaltspunkte haben. Die pompejanische Wasserleitung war ein $Z$ weig des großen Aquaedukts, der Neapel und die anderen Städte Campaniens mit Wasser versah. Ihr Ausgangsspunkt ist noch nicht festgestellt, das einzige, was sich über sie sagen läBt, ist, daB sie aus sehr großer Höhe kam und einen starken Druck hatte. Die Pfeiler des Aquädukts waren 6 Meter hoch. Fast an jeder Straßenachse befindet sich ein Brunnen. Die Wasserleitung in den Häusern ist am besten in dem Vícolo di Mercurio zu erkennen (s. S. 79). Da die Stadt auf einem Lava. felsen lag, so gab es nur wenige Quellen (s, S. 139).

Von der Kanalisation hat man einige Kollektoren (Sammelbecken) gefunden, zu denen die Rohre aus den Häusern führten. (Südende des Forums unter der Strada delle Scuole; neben den Stabianerthermen). Man erkennt deutlich, da $B$ die Kanalisation erst nach und nach in der Stadt eingerichtet wurde, in einigen Bezirken hat man noch Gruben für die Abwässer gefunden. Die meisten Latrinen haben noch keine Verbindung mit der Kanalisation; im Zusammenhang mit ihr steht z. B. die Latrine Regio IX, Insula 9.

$\mathrm{D}$ a s $\mathrm{H}$ a us. Wir können an dieser Stelle keine Geschichte der Entwicklung des antiken Hauses geben. Unser 
Ziel ist nur, dem Leser den Grundriß des pompejanischen Hauses klarzumachen und ihn mit den Benennungen der wichtigsten Räume vertraut $z u$ machen. Die Eigentümlichkeit der Entwicklung des pompejanischen Hauses liegt darin, $\mathrm{daB}$ es das Ergebnis der Verschmelzung zweier verschiedener Haustypen, von denen jedes seine eigene Geschichte hat, ist. Es entsteht aus dem italischen und griechischen Haus. Die ältesten Häuser haben rein italischen Grundriß. Aber als typisch für das pompejanische Haus muB man doch das Doppelhaus bezeichnen. Das alte italische Haus, das wir aus Aschenurnen kennen, bestand aus einem Raum, der den Bedürfnisssen der Zeit genügte. In der Mitte stand der Herd. Der aufsteigende Rauch färbte die Wände und das Dach schwarz, weshalb der Raum den Namen Ater d. h. schwarz (französisch l'âtre) erhielt. Hier war Küche, Wohnraum, Arbeitsraum und Schlafzimmer. Im Laufe der Zeit baute man um das Atrium herum für jeden $Z$ weck einen eigenen Raum. Mittelpunkt blieb aber immer das Atrium. Die Oeffnung im Dach. durch die im ersten Stadium der Rauch ging, blieb erhalten. wurde vergrößert und diente nun als Lichtquelle. Freilich fiel durch diese Oeffnung nicht nur das Licht in die Räume, sondern auch der Regen und daher belsam sie den Namen Compluvium. Dem Compluvium entspricht auf dem Fußboden das Impluvium, an dem nun immer ein Tisch steht, eine Erinnerung an den ehemaligen Standort des Herdes.. Selbst zu der Zcit, als man in allen Häusern schon Wasserleitungen hatte. hat man das Regenwassser gesammelt, was man aus der Anlage von $Z$ isternen schlieBt. Neben den Tischen, einfachen und reich verzierten aus Tuff und Marmor, stand auf einer Basis meist eine Statue mit Springbrunnen. Auch das Compluvium wurde verziert, an den Ecken waren meistens Tierfiguren als Wasserspeier angebracht und Maskenfriese an den Seiten.

Es gibt mehrere Arten von Atrien, am häufigsten sind in Pompeji das tuskanische (Atrium tuscanicum) und das viersäulige (Atrium tetrastylum). Im $t$ u s $\mathrm{k}$ a $\mathrm{n}$ is $\mathrm{ch}$ e A trium ruht das Compluvium auf den Horizontalbalken, es hat keine Säulen. Das Compluvium des A tri um te tra stylum ruht aut vier Säulen. Anstatt Rekonstruktionszeichnungen zu geben, die doch nicht immer allen An. forderungen entsprechen, verweisen wir auf die Wiederherstellungen in Pompeji selbst (mit Ausnahme der Häuser in den Nuovi Scavi, wo eine große Reihe von Atrien herge- 
stellt ist): A trium tu $\mathrm{s}$ canicum Casa dell'ara massima. (VI, 16, 16) und Casa di Lucrezio Frontone $(\mathrm{V}, 4,10)$; A t $\mathrm{r}$ i u m tetrasty lum Casa delle nozze d'argento $(\mathrm{V}, 2)$ und das Haus VI, 15, 9, dies ist besonders interessant dadurch, weil h:er auch die Räume des ersten Stockes wiederhergestellt sind. $Z_{w e i}$ Atrien sind sog. $\mathrm{korinth}$ is che Atrien, das eine befindet sich in der Casa di Castore e Polluce (VI, 9, 6) und das andere in der Casa di Epidio Rufo (IX, 1, 20). Ausnahmsweise kommt auch das Atrium ohne Complu. v I u $\mathrm{m}$ (Atrium testudinatum, d. h. schildkrötenförmig) $z$. B. im kleinen Hause $V, 5,2$ und im Nebenatrium in der Casa deı Centenario und ein Atrium mit darüberliegendem Raum in der Casa di Fabio Secondo $(V, 4,13)$ vor.

An den Seiten liegen die $\mathrm{Cubiculen.}$ Das sind kleine Räume, die als Schlafzimmer oder Räume für die Sklaven dienten. In den Schlafzimmern erkennt man den Platz, wo das Bett gestanden hat daran, daß sich hier entweder eine breite Stufe findet oder ein durch einen Mosaikstreifen bezeichneter Platz, einige Male sieht man auch in der dicken Wand eine Vertiefung, die man gemacht hat, um Platz für das Bett zu schaffen. Die letzten Räume an den Seiten, die Alae, die sich in ganzer Breite auf das Atrium öffnen, haben keine bestimmte Verwendung; einige Male dienen sie als Speisezimmer, andere Male als Hauskapelle (Sacellum).

Es gibt in Pompeji einige Häuser, in denen man von der Straße aus gleich das Atrium betritt (z. B. die kleinen Häuser in dem Vicolo di Lucrezio Frontone). Doch sind sie eine Ausnahme. Ueblich ist, daß die Atrien von der Straße durch Vestib ï I und Fauces getrennt sind. Das Wort vestibulum ist philologisch noch nicht befriedigend erklärt. Es war ein unbedeckter Raum vor der Tür, der in Rom in den Häusern der Reichen ziemlich groß war, in Pompeji dagegen sehr schmal ist und meistens nur aus einer Stufe besteht. Aber es gibt doch einige Häuser, in denen das Vestibül ganz klar von den Fauces getrennt ist (VI, 13, 13 und VIII, 2,34).

Die F a u ces sind ein Korridor zwischen zwei Türen, an dem meistens Läden liegen. Die Inschriften in den Fauces werden wir bei der Beschreibung der einzelnen Häuser erwähnen.

Die $\mathrm{Holzt}$ üren sind natürlicherweise verschwunden. Einige sind in den Nuovi Scavi wieder hergestellt. Im Museum gibt es einen Gipsabguß einer Tür. Zu einer klaren Vorstellung verhelfen uns außerdem noch die Marmortür 
an einem Grab in der Gräberstraße und vor dem Nucerinertor und die malerische Darstellung in der Villa Item.

Gegenüber dem . Eingang liegt das T a blin u m, das Empfangs- und Arbeitszimmer des Hausherrn. In einigen Häusern ist die Rückwand aus Brettern gewesen (tabulumBrett). $Z_{u}$ Seiten des Tablinums lagen Speisezinmer, die sich meistens auf das Peristyl öffneten. Das Tablinum war kein Durchgangsraum. Als Durchgang diente der And ron, der Atrium mit Peristyl verband. Das Wort Andron kommt aus dem Griechischen und heißt eigentlich Männerzimmer und warum diese Bezeichnung auf den Korridor übertragen wurde, läßt sich nicht erklären.

Wir kommen nun zu dem griechischen Teil des Hauses, was sich auch schon aus den Bezeichnungen ergibt. Das Peristyl (Säulen umher) ist ein innerer Hof von einer Portikus umgeben. Die Portikus braucht nicht auf allen vier Seiten vorhanden $z u$ sein, einige Male umgibt sie den Hof nur auf drei, andere Male nur auf zwei Seiten und es kommt auch vor, daB sie nur auf einer Seite entlang läuft. In einzelnen Häusern war die Portikus zweistöckig (Casa del Centenario [IX, 8, 6/], Casa del centauro [VI, 9, 3]). Das Peristyl war der malerischste Teil des Hauses. In ihm lag der Garten (Viridarium) mit Blumen, Statuen und Wasseranlagen reich verziert.

Dem Tablinum entspricht die Exedra, die eine Art Empfangszimmer war. Als Speisezimmer dienten die Tri. clinien. Der Name ist ganz leicht $z u$ erklären: hier standen drei Lager, auf denen Platz. für neun Personen war, die hier liegend speisen konnten. Diese Lager waren meistens aus Holz und reich verziert. Nicht selten findet man aufgemauerte Lager. Sie waren bemalt, auf einem in den Nuovi Scavi ist die Malerei fast ganz erhalten. Natürlich wurden noch Kissen auf sie gelegt. In einem Hause findet man auch cine Lagerstätte für Kinder (IX, 5, 11) und in einem anderen ein Lager in Form des Buchstabens Sigma (VIII, 3, 15).

Als Festsaal diente der O e cus. Philologisch ist auch diese Benennung nicht leicht zu erklären, weil Oecus ein. fach Haus bedeutet. In der Casa di Meleagro und del Labe. rinto finden wir solche Räume mit einer Säulenstellung im Innern. In dem Innenraum standen die Lagerstätten und der Gang hinter den Säulen war für die bedienenden 
Sklaven bestimmt, die an dem Lager zu Füßen ihrer Herren standen. Ein ganz besonderer Oecus befindet sich in der Casa delle Nozze d'argento (s. S. 115).

In einigen Häusern hat man die Schlafzimmer der Familie auch an das Peristyl angebaut. So entwickelte sich das Peristyl und die anliegenden Räume zu einem intimeren Teil des Hauses und das Atrium mit seinen Räumen wurde Geschäftsraum.

Von den meisten Häusern waren Teile vermietet. Gewöhnlich war das der Fall mit dem Oberstock, dessen Existenz schon durch die Treppen bewiesen wird (von den Treppenanlagen wird jeweils an ihrem Platz die Rede sein). Einige Häuser haben im Oberstock einen Erker und andere sog. Cenacula, eine Art Loggia, die als Speiseraum diente.

Einen ganz anderen Anblick bekamen die Ruinen von Pompeji, nachdem die Direktion der Ausgrabungen die Wiederherstellung der Gärten in Angriff genommen hatte. Man hat sorgfältig jede Pflanzenspur aussgenutzt und sich nach de:l malerischen Vorbildern an den Wänden gerichtet. Besonders gut gelungen ist die Wiederherstellung des Gartens VIII, 7, 5 (s. S. 192), die größeren Häuser hatten außer dem Garten im Peristyl meist noch einen zweiten Garten.

Zum Schluß erwähnen wir noch das Posticum, den Hintereingang, das jedes Haus besaß.

Die weitverbreitete Meinung, daB die Pompejaner nur Wert auf die Ausschmückung des Inneren ihres Hauses legten und das Aussehen der Fassade ihnen gleichgültig war, ist ganz grundlos. Nicht grau und düster sahen die Straßen Pompejis aus, sondern bunt und heiter. Der Besucher wird an verschiedenen Stellen noch Reste von Fassadenmalerei bemerken. Die Wände der Häuser in den Nuovi Scavi sind von oben bis unten mit Wahlprogrammen bedeckt. Das gleiche war auch in den anderen Teilen der Stadt der Fall, nur sind hier die Inschriften größtenteils verblaßt. Aus ihnen läßt sich noch lebendig die Heftigkeit des Kampfes um den Posten des Duumvir und des Aedilen erkennen, der höchsten Verwaltungsstellen der Städte, die Selbstverwaltungsrecht besaßen.

Die schmalen Fenster geben den Häusern fast ein blindes Aussehen. Aber das Straßenbild wird doch belebt durch die Läden. AuBerdem haben wir auch breitere Fenster in der Stadt, z. B. in der Casa di Lucrezio Frontone und 
im ersten Stock der Bäckerei an der Strada di Stabiae (VIII, 4, 24).

Wir haben hier nur die allgemcinen Grundzüge des Hauses beschrieben. Selbstverständlich hat jedes sein eigenes Gesicht und gerade darin besteht der Reiz Pompejis.

Die M a lerei. Zu dem Wichtigsten und Schönsten in der Stadt gehört die Wandmalerei. Die Archäologen haben das System von Mau angenommen, das sehs zur Orienticrung in der großen Menge der erhaltenen Fresken hilft. Mau teilt die Malerei in vier Stile, die natürlich nicht immer ganz scharf abzugrenzen sind.

I. Stil. Hier hatte der Stuckateur mehr zu tun als der Maler. Man ahmte in Stuck die Marmorquadern nach und strich sie mit den verschijedensten Farben, an. In diesem Stil findet man keine Bilder, die Bilder findet man in den FuBbodenmosaiken. Oben schlieBt die Bemalung ein Zahnschnitt aus Stuck ab. Beispiele: Casa di Sallustio, Casa del Fauno, Casa delle Amazzoni (VI, 2, 14), Schlafzimmer in der Casa del Centauro, Schlafzimmer im Hause VI, 5, 19, ein Laden in der Stabianerstraße (IX, 3, 2). Diese Malerei gehört noch der vorrömischen Zeit an.

II. Stil. Keine Stuckarbeiten mehr. Nachahmung von Architekturformen, körperlich wirkende Säulen und vorspringende Gebälke. Zwischen den Säulen Girlanden, Erstes Auftreten von Bildern, die sich in die Architekturmotive eingliedern. Beispiele: Casa del Laberinto, Casa di Babio Rufo (VII. 2. 16), Casa di Obellio Firmo, Villa Item. Zeit der römischen Republik.

III. Stil, Die architektonischen Motive werden nicht mehr impressionistisch, sondern zeichncrisch und flach dargestellt. Besondere Sorgfalt wird der Ornamentierung gewidmet. Die rechteckigen Felder zwischen den Architekturmotiven schmücken Bilder mit mythologischen Scenen, mit Tieren und Landschaften. Am einheitlichsten findet man diesen Stil im Atrium und Tablinum in der Casa di Lucrezio Frontone. In der Stadt hat sich außerdem nur noch ein besonders typisches Beispiel in der Casa di Caecilio Giocondo erhalten. Erste Kaiserzeit bis etwa $60 \mathrm{n}$. Chr.

IV. Stil. Die meisten Forscher sehen in diesem Stil nicht die Weiterentwicklung des dritten Stiles, sondern des zweiten. Phantastische Architekturen mischen sich mit re- 
alen, wieder impressionistisch gesehenen Motiven. Sehr beliebt sind Durchblicke ins Freie, Loggien und Pergolen. Ganz unorganisch sind die Bilder eingelassen: mythologische Scenen und fliegende Figuren. Dieser Stil ist natürlich der häufigste, da nach der Katastrophe von 63 fast alle Häuser in ihm geschmückt wurden. Das typischste Beispiel mit allen Mängeln und Vorzügen dieses Stils ist die Casa dei Vettii.

Nach alter Tradition sind am Son $\mathrm{tag}$ eine große $\mathrm{Zahl}$ der interessantesten, reich bemalten Häuser geschlossen und den eindringlichsten Vorstellungen wird es nicht gelingen, diesen Brauch zu durchbrechen. So sieht man an diesem Tag eine Menge enttäuschter Besucher. Nun kann man freilich diesen $\mathrm{Tag} z u$ einer allgemeinen Orientierung in großem Maße ausnutzèn, und bei der Ausdehnung der Ruinen ist es noch fraglich, ob dieser eine Tag für ein eingehenderes Studium genügt, doch muB man sagen, daß derjenige Besucher, der Pompeji nur am Sonntag sah, 'sehr viel wichtige Dinge nicht kennengelernt hat.

Wir werden im folgenden versuchen, den Besucher an einem Sonntag Schritt für Schritt durch die Stadt zu führen, ob Zeit und Kraft für die Durchführung dieses Planes ausreichen, müssen wir ihm selbst überlassen. Von den Tempeln sind der Apollotempel, der Isistempel und der Tempel des Zeus Meilichios geschlossen, aber von allen dreien kann man einen ersten Eindruck bekommen. Von den öffentlichen $\mathrm{Ge}$ bäuden sind nur das Macellum und die Thermen geschlossen. Alle Werkstätten, viele Häuser, und die Gräberstraße sind zugänglich, also mehr wie genug für einen Tag.

Der Eintritt ist unentgeltlich.

Man betritt die Stadt wie immer an der Porta Marina. Man geht direkt zum Forum und betrachtet: die Basilika, Curien, Comitium, Gebäude der Eumachia, Vespasianstempel, Heiligtum der städtischen Laren, Jupitertempel, die Basen mit Inschriften aut dem Forum: besteigt die Treppe, von der aus man den Apollotempel gut übersehen kann und verläßt das Forum durch die Straße links vom Jupitertempei und kommt in die Via dei Soprastanti. Man geht in die Via delle Terme: rechts Schild eines Stadtquartiers mit der Ziege, links Thermopolium mit besonders gut erhaltenem Tonrohr. Weiter links Wasserreservoir und gegenüber der kleine Hof der Forumsthermen. Man überschreitet die Strada di Nola und besucht die Casa di Pansa. Man verläBt das 
Haus durch das Posticum und geht gegenüber in die gut erhaltene Bäckerei und Mühle. Durch sie hindurch gehend gelangt man in die Strada consolare und betrachtet die Casa di Sallustio. An der Straßengabelung ein Brunnen, dann das sog. Zollamt und die Casa del Chirurgo. Herkulanertor und Gräberstraße (Villen geschlossen). Zurück zum Herkulanertor und durch die Casa delle Vestale (links) in den Vicolo di Narzisso. Man besucht das Haus Nr.14 (Casa delle Amazzoni) und geht in das Haus Nr. 15 (delle danzatrice). Im Peristylium rechts sieht man ein ganz besonders schönes Lararium. Durch dieses Haus kommt man in den Vicolo modesto. Man besucht hier die Casa di Nettuno (VI , 5, 3): an der Wand rechts eine Tholos; auBerdem die Darstellung eines Gespannes und aut den FuBböden grobe aber typische Mosaiken. Im Hause Nr. 5 befindet sich im Peristyl ein gut erhaltener Stuckfries. In einer kleinen Kammer sieht man die Trommeln einer bemalten Säule (s. S. 63). Man kommt nun in den Vicolo della Fullonica. Etwas links sieht man eine große Oeffnung in der Wand, durch die man sehr gut den Garten der Casa di Apollo sehen kann. Im Hause Nr. 19 befindet sich ein schönes im ältesten Stil bemaltes Schlafzimmer. Wir sind jctzt im Vicolo di Mercurio. Links sieht man eine bemalte Außenwand. Iiast alle Häuser an der Strada di Mercurio sind geschlossen, geöffnet sind an der linken Seite die Casa di Inaco ed Io und dell'argenteria. An der rechten Seite ist die Casa di Centauro geöffnet, außer dem Schlafzimmer, das immer geschlossen ist. Das Posticum führt uns in den Vicolo del Fauno. Der Besucher spart Zeit, wenn er durch clas Haus Nr. 6 in den Vicolo del Laberinto geht. Im Vicolo del Laberinto besucht man das Haus VI, 15, 23 (nur mit Peristyl und besonders schönem Lararium). Man geht durch den Einging Nr. 24 und kommt aus dem Hause Nr. 5 in den Vicolo dei Vettii (das Haus hat ein Sacellum mit drei Nischen). In dieser Gasse sind auf der gegenüberliegenden Seite folgende Häuser geöffnet (VI, 16) 32 (über der Tür sieht man zwei Nischen für die Penaten, in dem kleinen Zimmer links eine Landschaft mit dem heiligen Baum. Im Hause Nr. 27 im Peristyl auf dem Sockel groBe Vögel; beachtenswert sind groBe Dolien. Castell d'aqua ist geschlossen. Wir sind jetzt auf der Strada di Stabiae. In diesem Teil ist nur die Westseite ausgegraben. Im Haus Nr. 18 horizontal gelegte Amphoren. Durch das große Fenster sieht man ganz gut das Atrium der Casa dell'ara massima. Im Haus Nr. 12 Masken und Lararium 
(S. 102). Wir gehen an einer Reihe geschlossener Häuser vorbel bis zur Ecke der Via di Nola. Die Zentralthermen sind geöffnet. Nun folgen einige Thermopolien und Bärkereien, dann das kleine Haus $V, 3,15$ mit 'Triclinien. In dem Vicolo di Lucretio Frontone sind fast alle Häuser geöffnet, auch die beiden Häuser, die hinter der Casa di Lucretio Frontone liegen. Die Häuser in der nächsten Gasse sind alle geöffnet. Jetzt Porta di Nola und Gräber. Nun zurück. An der linken Seite liegt die Casa di Obellio Firmo mit den Skeletten im Vestibül (geschlossen). Weiter im Haus IX, 9, 1 die gut erhaltene Treppe. An der Casa del Centenario vorbei in die Gasse links (Vicolo del fauno ubriacco). Am Ende der Gasse eine ganz besonders bunte Nische (IX, 6, 7). Nun die Parallelgasse (Vicolo di Lucrezio) zur Strada di Nola bis zur nächsten Ecke: Vicolo di Tesmo; an einem Hause eine bemalte Außenwand und ein gut erhaltenes Wahlprogramm; und wieder links zur Bäckerei und Caupona IX, 3, 17/20. Das Haus Nr. 24 ist ein Fuhrleutegasthaus mit dem Bilde der Göttin Epona. So sind wir wieder auf der Strada di Stabiae. Links sehen wir ein Schild (IX, 2, 7) mit Instrumenten. An der linken Seite der Strada di Stabiae liegt die Gerberei (s. S. 212), das Haus mit Compluvium (s. S. 211) und das Lararium mit den Schlangen in Stuck (s. S. 212). Nun Stabianertor und Gräber (s. S. 210). Zurück und zum kleinen Theater und dem ganzen zu dem Theaterbezirk gehörigen Komplex (S. 196). Durch die Propyläen in die Strada dei Teatri und nach Ueberschreitung der Strada dell'Abbondanza in das Labyrinth der kleinen Gassen. Links im Vicolo di Lupanaro liegt eine große Herberge (VII, 11, 14). Man kommt auf die Straße degli augustali. In diesem Bezirk ist die Orientierung nicht so leicht, man beachte, daB der Vicolo del panettiere auf die Strada di Stabiae und der Vicolo storto auf die Strada di Nola führt. Man biegt jetzt links in die Strada di Nola ein und gelangt an einigen geschlossenen Häusern vorbei zu der Casa degli capitelli figurati (VII, 4, 57 S. 136). Man kann noch einige Schritte weiter die Strada di Nola entlang gehen bis zum Tempel der Fortuna und dann wieder zurück zur Casa delle parete nere (S. 134) und dann durch die Casa degli capitelli figurati und die Konditorei (S. 135) und überschreitet die Via degli augustali und geht durch das Haus $\mathrm{Nr}$. 30, wo man auch ein Fresko mit Gartendarstellungen sieht und nach Ueberquerung der engen Gasse kommt man in die Casa delle Nozze di Ercole (S, 147). Nun durch die Via di Eumachia 
und nach Ueberschreitung der Via dell'Abbondanza in den Vicolo dei 12 Dei. Links VIII, 5, 9 Bäckerei mit Marmorfußboden und ein Keller. Dann weiter bis zur Via della Regina, der letzten Straße am Südteil, hier sind links die Häuser Nr. 34, 28, 26, rechts Nr. 15 (Haus mit Sigma), Eckhaus Nr. 21 geöffnet. Dic Via delle Scuole führt zum Südteil des Forums. 
